\title{
A Wearable Gait Analysis System Used in Type 2 Diabetes Mellitus Patients: A Case-Control Study
}

\author{
Tian Tian (D) \\ Cheng Wang ${ }^{2-4}$ \\ Yuan $\mathrm{Xu}^{5}$ \\ Yuzhi Bai ${ }^{1}$ \\ Jing Wang' \\ Zhou Long ${ }^{2,3}$ \\ Xiangdong Wang ${ }^{6,7}$ \\ Lichun Zhou ${ }^{8}$
}

'General Practice Department, Beijing

Chao-Yang Hospital, Capital Medical

University, Beijing, People's Republic of

China; ${ }^{2}$ Institute of Digital Economy

Industry, ICT, Hangzhou, People's

Republic of China; ${ }^{3}$ Luoyang Institute of Information Technology Industries, Luoyang, People's Republic of China;

${ }^{4}$ Ningbo Institute of Information Technology Application, CAS, Ningbo, People's Republic of China; ${ }^{5}$ Department of Endocrinology, Beijing Chao-Yang Hospital, Capital Medical University, Beijing, People's Republic of China; 'Institute of Computing Technology (ICT), Chinese Academy of Sciences (CAS), Beijing, People's Republic of China; ${ }^{7}$ Beijing Key Laboratory of Mobile Computing and Pervasive Device, Beijing, People's Republic of China; ${ }^{8}$ Department of Neurosurgery, Beijing Chao-Yang Hospital, Capital Medical University, Beijing, People's Republic of China
Correspondence: Yuan Xu

Email xuyuan3200@sina.com
Background: Previous studies have shown that the gait of patients with type-2 diabetes mellitus is abnormal compared with the healthy group. Currently, a three-dimensional motion analyzer system is commonly used for gait analysis. However, it is challenging to collect data and use in clinical study due to extensive experimental conditions and high price. In this study, we used a wearable gait analysis system (Gaitboter) to investigate the spatial and temporal parameters, and kinematic data of gait in diabetic patients, especially those with peripheral neuropathy. The aim of the study is to evaluate the wearable gait analysis system in diabetic study.

Materials and Methods: We conducted a case-control study to analyze the gait of type 2 diabetes mellitus. Gaitboter was used to detect and collect gait data in the ward of Beijing Chao-yang Hospital, Capital Medical University from June 2018 to October 2018. We collected the gait data of participants $(\mathrm{N}=146 ; 73$ patients with type 2 diabetes, 16 with peripheral neuropathy and 57 without peripheral neuropathy, and 73 matched controls). The gait data (stance phase, swing phase, double-foot stance phase, single-foot stance phase, walking cadence, stride length, walking speed, off-ground angle, landing angle, maximum swing angle, minimum swing angle, and foot progression angle) in diabetic patients were recorded and compared with controls. SPSS 22.0 statistical software was used to analyzed the gait parameter data.

Results: We found that the landing angle and the maximum swing angle of diabetes patients with or without peripheral neuropathy were significantly less than those of the control group $(\mathrm{P}<0.05)$. The walking speed of diabetes patients with peripheral neuropathy is significantly less than those of the control group $(\mathrm{P}<0.05)$.

Conclusion: This study confirms that the wearable gait analysis system (Gaitboter) is an ideal system to identify abnormal gait in patients with type 2 diabetes and provides a new device and method for diabetes-related gait research.

Keywords: gaitboter, gait analysis, diabetes mellitus, peripheral neuropathy, swing phase

\section{Introduction}

Diabetic peripheral neuropathy (DPN) is asymmetrical, length-dependent sensorimotor polyneuropathy related to metabolic and microvessel changes due to hyperglycemia and, consequently, cardiovascular risk. ${ }^{1}$ The onset of additional diabetic microvascular complications in the person confirms that DPN is due to diabetes. The incidence of DPN in diabetes may be as high as $50 \%$ based on age and extent of diabetes, ${ }^{2}$ and symptomatic painful DPN can affect up to $30 \%$ of diabetic patients with neuropathy ${ }^{3}$ Generally, DPN signs could be either positive (aching, swelling, acute or pressure pain) or negative (a numbness or a dead feeling) or both at the same 
time. ${ }^{4}$ Since DPN affects the visual, motor and autonomic components of the nervous system, which manifests itself as a lack of protective sensation, intestinal foot muscle dysfunction may contribute to the alteration of the gait. ${ }^{5}$ Previous studies have shown that the gait of patients with type-2 diabetes mellitus, especially those with peripheral neuropathy is abnormal compared with healthy individuals. ${ }^{2,6}$ Furthermore, studies also confirmed that diabetic patients have abnormal gait before the onset of feet sensory impairment. ${ }^{6-8}$ Currently, the commonly used threedimensional motion analyzer system for gait analysis can only describe the gait under laboratory conditions. It usually requires a combination of multi-camera motion capture system and force platforms. ${ }^{3}$ It is challenging to collect data and use in clinical study due to extensive experimental conditions and high price. In addition, employing force platforms or film-pressure sensors' console for gait analysis cannot appropriately study the swing phase. ${ }^{9}$ The new wearable measurement method mainly relies on inertial sensors that can combine accelerometer and gyroscope. ${ }^{10}$ The corresponding positions' motion information can be obtained by mounting the above sensors in the key parts of lower limbs. However, this method has several challenges. ${ }^{11}$ Therefore, in the study, we used the portable and wearable gait analysis system (Gaitboter) developed by the Institute of Computing Technology, Chinese Academy of Sciences to analyze the gait of diabetic patients. ${ }^{12-14}$ The Gaitboter is simple to operate, and the results are accurate. ${ }^{14}$ A previous study has confirmed that it has better sensitivity and stability for the evaluation of walking ability. ${ }^{15}$ This study used the portable and wearable gait analysis system (Gaitboter) to analyze the gait of type 2 diabetic patients for the first time, and explore the application value of wearable gait system in diabetic diseases.

\section{Materials and Methods Study Design}

We conducted a case-control study to analyze the gait of type 2 diabetes mellitus. Gaitboter, a portable and wearable gait analysis system developed by Institute of Computing Technology, Chinese Academy of Sciences, was used to detect and collect gait data in the ward of Beijing Chao-yang Hospital, Capital Medical University.

\section{Protocol/Data Collection}

All participants were asked to wear the suitable size device, walk for $10 \mathrm{~m}$ at an optional speed in comfortable condition on a straight road without obstacles. From June 2018 to October 2018, we collected the gait data of 16 diabetic patients with peripheral neuropathy (DPP) [mean age 60.5 (56-67) years, mean body mass index (BMI) 26.96 (24.26-28.58) kg/m² , and meantime since diabetes diagnosis $15.96 \pm 7.57$ years] and 57 diabetic patients without peripheral neuropathy (DNP) [mean age 57 (48-65) years, mean body mass index (BMI) $25.46(23.51-27.57) \mathrm{kg} / \mathrm{m}^{2}$, and time since diabetes diagnosis $9.97 \pm 6.73$ years] hospitalized in Beijing Chao-yang Hospital. Although research has not firmly established causal relationships for gait changes in patients with diabetes, peripheral neuropathy remains suspect number one. ${ }^{16}$ Therefore, in the current study, we evaluate the gait data of diabetic patients with or without peripheral neuropathy. We also collected the gait data of healthy individuals without diabetes [mean age 53 (44-62) years, mean body mass index (BMI) $\left.24.24(23.15-26.46) \mathrm{kg} / \mathrm{m}^{2}\right]$, who were inhabitants of Beijing recruited sequentially into the study, from June 2018 to October 2018 and serve as the control group (CG). The subjects in the three groups (DPP, DNP, and CG) were matched in sex ratio, height and body mass index (BMI) to avoid selection bias as far as possible. Meanwhile, the subjects' height, weight, and time since diabetes diagnosis were collected. Blood samples of diabetic mellitus patients were collected for glycated hemoglobin (HbA1c), glycated albumin (GA).

\section{Inclusion and Exclusion Criteria}

The study includes patients diagnosed with type 2 diabetes and met the diagnostic criteria issued by the World Health Organization (WHO) in 1999. ${ }^{17}$ Peripheral neuropathy was confirmed by the Michigan Neuropathy Screening Instrument (MNSI) scale. $^{18}$ MNSI scale includes a questionnaire and bipedal physical examination. In this study, the diagnostic cut-off point of MNSI was that the questionnaire score is 4 and the physical examination score is $2{ }^{18}$ Patients were excluded from the study who were unable to walk independently, lower limb amputation (toe) history, obvious foot deformity, lower limb ulcer, brain injury history, poor vision affecting walking, various spinal diseases, patients with severe liver and kidney insufficiency, pregnancy and diabetes acute complications.

\section{Ethical Approval}

The study was approved by the institutional review board of Beijing Chao-yang Hospital, Beijing China, and was conducted in accordance with the Declaration of Helsinki. All participants have signed informed consent forms. The 
study protocol was submitted and approved by the Research Ethics Committee of the Beijing Chao-Yang Hospital (the number is 2018-sci-153). Institutional Review Board reference date is 2018-6-11.

\section{Methods and Equipment}

We used a Gaitboter, a portable and wearable gait analysis system developed by the Institute of Computing Technology, Chinese Academy of Sciences. The system consists of a gait acquisition device and a tablet computer, as shown in Figure 1. The device is a wearable gait acquisition device (shoes) that integrates multimodal data collection sensors, such as film pressure, microphone, accelerometer and gyroscope sensors.

This algorithm is just for the handling of collected gait raw data by the tablet computer, and for actual use of the system, the algorithm was implemented by the application which can install into the tablet computer with operation system. Therefore, the algorithm input data are obtained from the presented prototype device, and the gait analysis software includes the corresponding gait raw data handling algorithm. Gaitboter system has been validated against the three-dimensional motion analyzer system by former researches that achieved high consistency and stability. ${ }^{10,14 .}$

\section{Experiment Site}

The experiment was conducted in the ward of Beijing Chao-yang Hospital, Capital Medical University. The test area was well lit, and the ground was flat without any obstacle.

\section{Gait Measurement}

The person who does the test was a specially trained physician with clinical experience. After obtaining informed consent, demographic data of gender, age, height, weight, shoe size, time since diabetes diagnosis. All participants were asked to wear the suitable size device (Figure 2) and walk

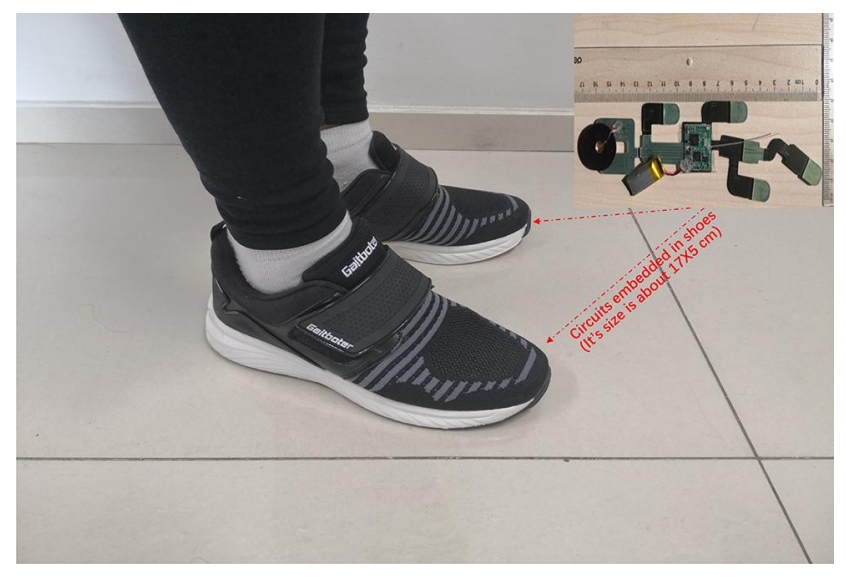

Figure 2 Pictures of wearing equipment for testing.

for 10- $\mathrm{m}$ at an optimal speed in a comfortable condition in a straight corridor free from obstacles. While walking, the tablet computer carried by the tester can synchronously receive walking data from the wearable devices through wireless Bluetooth connection. When the participant finished one time's test, his/her gait parameters will be computed automatically and shown on the tablet computer screen. Further methodology on how gait parameters were derived has been previously extensively described. ${ }^{12-14}$ The above three groups' gait parameters were analyzed statistically to evaluate the characteristics of gait parameters of diabetic patients. Meanwhile, the value of wearable gait analyzer in clinical research of diabetes mellitus was discussed.

\section{Observation Indicators}

In this part, several gait parameters were defined as the observation indicators (Table 1). Before giving the definition, the gait cycle should be explained at first. Gait cycle is defined as the time period during walking in which onefoot heel touches the ground when the same foot heel again touches the ground.

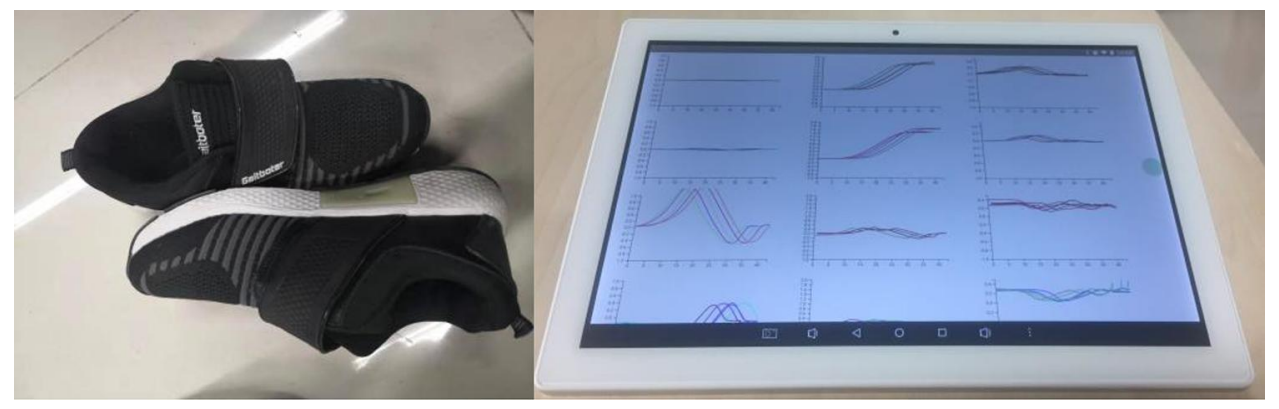

Figure I Picture of the system (gait acquisition device + tablet computer). 
Table I Various Observation Indicators with Their Units and Definitions

\begin{tabular}{|c|c|c|c|}
\hline $\begin{array}{l}\text { S/ } \\
\text { No }\end{array}$ & Indicators & Units & Definition \\
\hline I & Stance phase & s & $\begin{array}{l}\text { It is the time period during walking in which one-foot heel touch the ground to the time when the } \\
\text { same foot toe is off the ground }\end{array}$ \\
\hline 2 & Swing phase & s & $\begin{array}{l}\text { It is the time period during walking in which one-foot toe is off the ground to the time when the } \\
\text { same foot heel contacts the ground }\end{array}$ \\
\hline 3 & Double-foot stance phase & s & It is the time period when both feet are in contact with the ground during one gait cycle. \\
\hline 4 & Single-foot stance phase & s & It is the time period when one foot is in contact with the ground during one gait cycle. \\
\hline 5 & Walking cadence & Steps/min & It is step number per minute. \\
\hline 6 & Stride length & $\mathrm{m}$ & It is defined as the distance between any two successive points of heel contact of the same foot \\
\hline 7 & Walking speed & $\mathrm{m} / \mathrm{s}$ & It is the speed of walking. \\
\hline 8 & Off-ground angle & $\circ$ & It is the angle between the foot and the ground when the tip of the foot is off the ground. \\
\hline 9 & Landing angle & $\circ$ & It is the angle between the foot and the ground when the heel touches the ground. \\
\hline 10 & Maximum swing angle & $\circ$ & $\begin{array}{l}\text { It is the maximum angle in one gait cycle between the foot and the ground when the tip of the } \\
\text { foot towards up. }\end{array}$ \\
\hline 11 & Minimum swing angle & $\circ$ & $\begin{array}{l}\text { It is the maximum angle in one gait cycle between the foot and the ground when the tip of the } \\
\text { foot towards down. It is negative due to direction. }\end{array}$ \\
\hline 12 & Foot progression angle & $\circ$ & It is the angle between the long axis of the foot and the direction of the step. \\
\hline
\end{tabular}

\section{Statistical Analysis}

SPSS 22.0 software was used for statistical analysis. Differences of the time since diabetes diagnosis, HbA1C, GA between diabetic patients with peripheral neuropathy and without peripheral neuropathy were tested by independent samples $t$-test. The mean values of three groups' raw data and gait parameters were compared using One-way ANOVA, with post-hoc comparisons where significant main effects are found. Gender was compared by chisquare testing. A P-value $<0.05$ was considered as statistically significant difference.

\section{Results}

\section{Clinical Data Analysis}

The clinical data of the experimental subjects were analyzed, and the results are shown in Table 2 and Figure 3. There is no statistical difference in sex ratio, height, age, and BMI between the three groups $(\mathrm{P}>0.05)$. Diabetic patients with peripheral neuropathy have longer time since diabetes diagnosis than diabetic patients without peripheral neuropathy $(\mathrm{P}<0.05)$. There is no statistical difference in $\mathrm{HbA1C}$, and GA between the DPP group and the DNP group $(\mathrm{P}>0.05)$.

\section{Gait Parameter Analysis}

As shown in Table 3 and Figures 4-6, the landing angle and the maximum swing angle of diabetes patients with or without peripheral neuropathy are significantly less

Table 2 Comparison of Clinical Data in Three Groups

\begin{tabular}{|l|l|l|l|l|}
\hline Item & $\begin{array}{l}\text { DPP } \\
\text { Group } \\
(\mathbf{n}=16)\end{array}$ & $\begin{array}{l}\text { DNP } \\
\text { Group } \\
(\mathbf{n}=57)\end{array}$ & $\begin{array}{l}\text { CG Group } \\
(\mathbf{n}=73)\end{array}$ & P-value \\
\hline Female/Male & $9 / 7$ & $32 / 25$ & $38 / 35$ & 0.883 \\
\hline Age (years) & $58.88 \pm 7.90$ & $55.07 \pm 11.43$ & $52.23 \pm 12.43$ & 0.088 \\
\hline Height (m) & $1.66 \pm 0.07$ & $1.68 \pm 0.08$ & $1.66 \pm 0.08$ & 0.641 \\
\hline BMI (kg/m2) & $26.81 \pm 3.79$ & $25.46 \pm 3.82$ & $25.18 \pm 3.34$ & 0.191 \\
\hline $\begin{array}{l}\text { time since } \\
\text { diabetes diagnosis } \\
(y)\end{array}$ & $15.96 \pm 7.57$ & $9.97 \pm 6.93$ & & $0.004 *$ \\
\hline HbAIC (\%) & $9.21 \pm 2.14$ & $9.64 \pm 2.10$ & & 0.474 \\
\hline GA(\%) & $25.96 \pm 8.12$ & $25.99 \pm 7.72$ & & 0.987 \\
\hline
\end{tabular}

Notes: “*” of $P$ is less than 0.05 .

Abbreviations: DPP, diabetic patients with peripheral neuropathy; DNP, diabetic patients without peripheral neuropathy; CG, control group; BMI, body mass index; $\mathrm{HbAlc}$, glycated hemoglobin; GA, glycated albumin. 


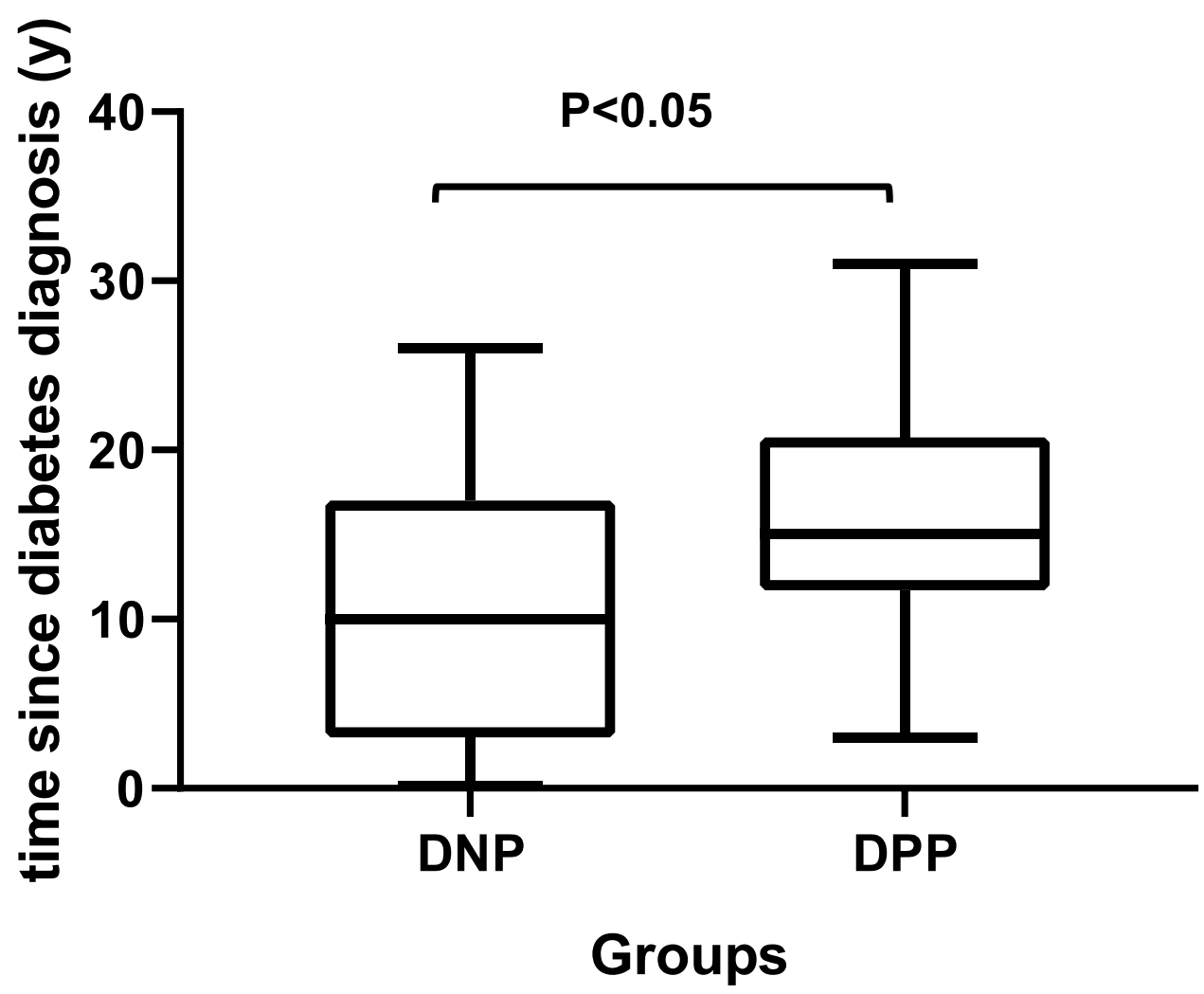

Figure 3 Mean of time since diabetes diagnosis $(y)$ in different groups.

Abbreviations: DPP, diabetic patients with peripheral neuropathy; DNP, diabetic patients without peripheral neuropathy.

than those of the control group $(\mathrm{P}<0.05)$. There are no statistical differences in the landing angle and the maximum swing angle between diabetes patients with and without peripheral neuropathy $(\mathrm{P}>0.05)$. The walking speed of diabetes patients with peripheral neuropathy is significantly less than those of the control group ( $\mathrm{P}<$ 0.05). The walking speed of diabetes patients without peripheral neuropathy tends to decrease compared with the control group, but it did not show any statistical significance. There are no statistical differences in the walking speed between diabetes patients with and without peripheral neuropathy $(\mathrm{P}>0.05)$. There are no statistical differences in swing phase, double-foot stance phase, single-foot stance phase, stance phase, walking cadence, off-ground angle, minimum swing angle and foot progression angle between three groups $(\mathrm{P}<0.05)$. The stride length of the diabetes group is tending to decrease compared with the control group, but it did not show any statistical significance. The foot progression angle of the diabetes group is tending to increase compared with the control group, but it did not show any statistical significance.

\section{Discussion}

Previous studies reported that diabetic patients have an abnormal gait. ${ }^{6}$ Those with diabetic neuropathy are at a far higher risk of slipping than those with normal sensation. ${ }^{19}$ The fact that most falls occur throughout locomotion ${ }^{20}$ indicates that these patients may have trouble maintaining dynamic equilibrium while walking. Current studies mainly focus on the mechanism of diabetic foot ulcer development and the increasing risk of falling due to abnormal gait in patients with diabetes mellitus. ${ }^{21-23}$ These adverse effects have a serious impact on the quality of life and life expectancy of diabetic patients. According to the use of different gait detection instruments, previous researchers have studied the gait of diabetic patients from various perspectives, and the focus of their research is different. ${ }^{24,25}$ Most of the studies used force measuring board to study the plantar pressure of patients during walking, but the description of gait in the swing phase is insufficient. ${ }^{9,26}$ The three-dimensional gait analyzer describes the spatial and temporal parameters of gait more carefully and accurately; however, it is difficult to apply in the clinic due to its large size, high cost and 
Table 3 Comparison of Gait Parameters in Three Groups

\begin{tabular}{|c|c|c|c|c|c|c|}
\hline Item & DPP Group $(n=16)$ & DNP Group $(n=57)$ & CG Group $(n=73)$ & PI & $\mathbf{P 2}$ & $\mathbf{P 3}$ \\
\hline Stride length (m) & $1.26 \pm 0.19$ & $1.30 \pm 0.14$ & $1.34 \pm 0.11$ & 0.076 & 0.228 & 0.511 \\
\hline Double-foot stance phase (s) & $0.14 \pm 0.03$ & $0.14 \pm 0.03$ & $0.13 \pm 0.03$ & 0.226 & 0.221 & 0.834 \\
\hline Stance phase $(s)$ & $0.70 \pm 0.06$ & $0.69 \pm 0.03$ & $0.68 \pm 0.07$ & 0.285 & 0.344 & 0.816 \\
\hline Swing phase (s) & $0.4 I \pm 0.02$ & $0.4 I \pm 0.03$ & $0.40 \pm 0.04$ & 0.796 & 0.693 & 0.992 \\
\hline Single-foot stance phase (s) & $0.4 I \pm 0.02$ & $0.4 I \pm 0.03$ & $0.40 \pm 0.04$ & 0.812 & 0.720 & 0.992 \\
\hline Walking cadence (spm) & $|18.93 \pm 6.6|$ & $|10.7| \pm 9.11$ & $113.03 \pm 9.80$ & 0.246 & 0.334 & 0.775 \\
\hline Walking speed $(\mathrm{m} / \mathrm{s})$ & $1.15 \pm 0.22$ & $1.21 \pm 0.18$ & $1.27 \pm 0.16$ & $0.04 I^{*}$ & 0.107 & 0.503 \\
\hline Off-ground angle $\left({ }^{\circ}\right.$ ) & $-69.83 \pm 7.05$ & $-70.56 \pm 6.46$ & $-70.79 \pm 5.97$ & 0.976 & 0.834 & 0.912 \\
\hline Landing angle $\left({ }^{\circ}\right)$ & $13.59 \pm 4.77$ & $15.68 \pm 4.94$ & $18.35 \pm 5.35$ & $0.003^{*}$ & $0.011^{*}$ & 0.323 \\
\hline Maximum swing angle $\left({ }^{\circ}\right)$ & $16.15 \pm 4.01$ & $18.8 \mid \pm 4.63$ & $20.82 \pm 4.67$ & $0.00 I^{*}$ & $0.038^{*}$ & 0.105 \\
\hline Minimum swing angle $\left({ }^{\circ}\right)$ & $-70.70 \pm 7.04$ & $-71.54 \pm 6.48$ & $-72.64 \pm 6.02$ & 0.509 & 0.588 & 0.886 \\
\hline Foot progression angle $\left({ }^{\circ}\right)$ & $12.65 \pm 5.10$ & $11.90 \pm 4.47$ & $10.27 \pm 4.16$ & 0.125 & 0.093 & 0.819 \\
\hline
\end{tabular}

Notes: "**” of $P$ is less than 0.05 .

Abbreviations: DPP, diabetic patients with peripheral neuropathy; DNP, diabetic patients without peripheral neuropathy; CG, control group; PI, comparison between CG group and DPP group; P2, comparison between CG group and DNP group; P3, comparison between DPP group and DNP group.

complex mode of operation. Therefore, the gait analysis instruments applied in clinics on diabetes patients and scientific research are crucial. The portable and wearable gait analysis system (Gaitboter) developed by the Institute of Computing Technology, Chinese Academy of Sciences, is cheap, easy to operate and has low laboratory requirements. $^{12,13}$ These characteristics of Gaitboter can meet the clinical requirements. Moreover, the method
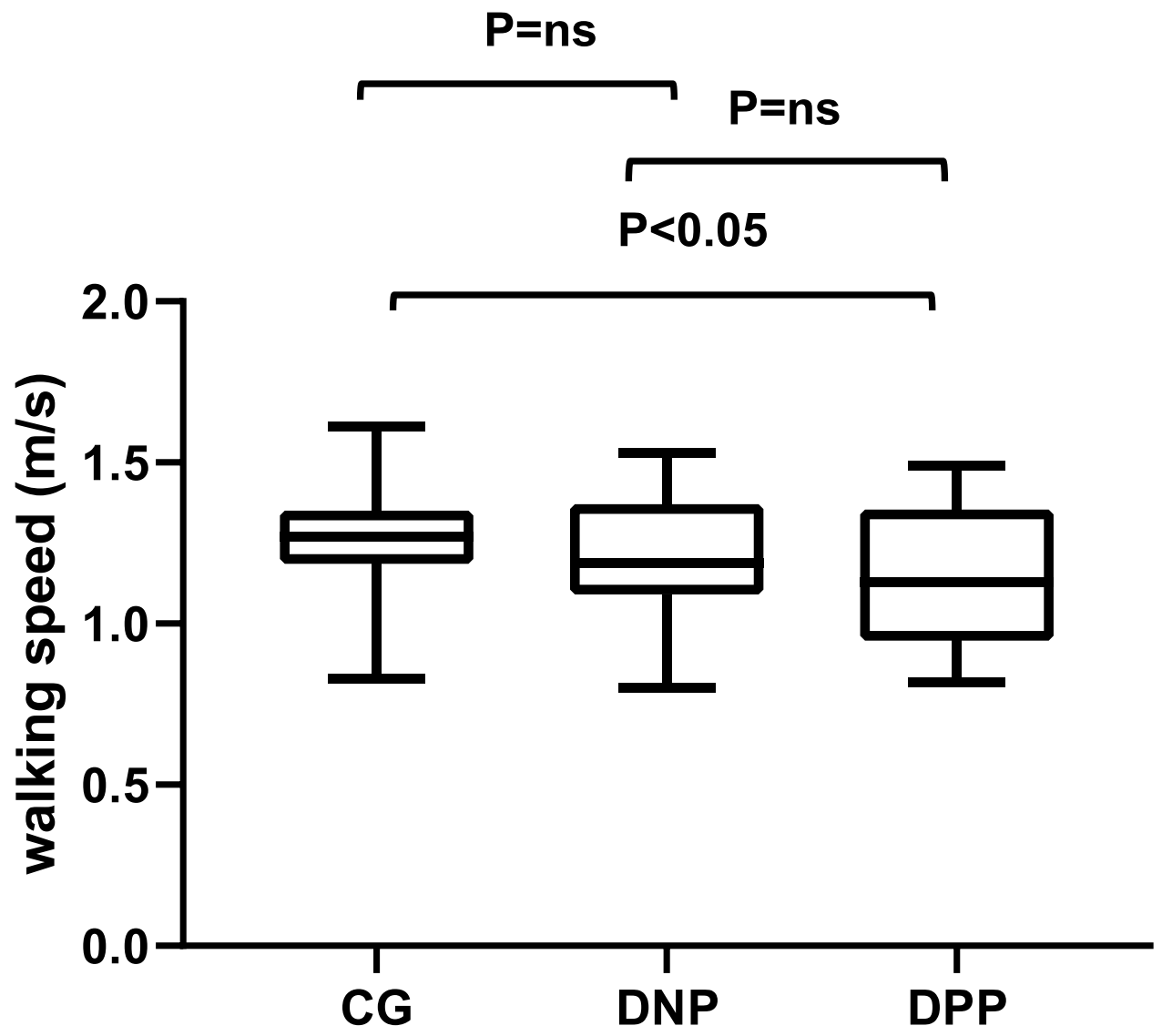

Figure 4 Mean of walking speed $(\mathrm{m} / \mathrm{s})$ in different groups.

Abbreviations: DPP, diabetic patients with peripheral neuropathy; DNP, diabetic patients without peripheral neuropathy; CG, control group. 


\section{P=ns}
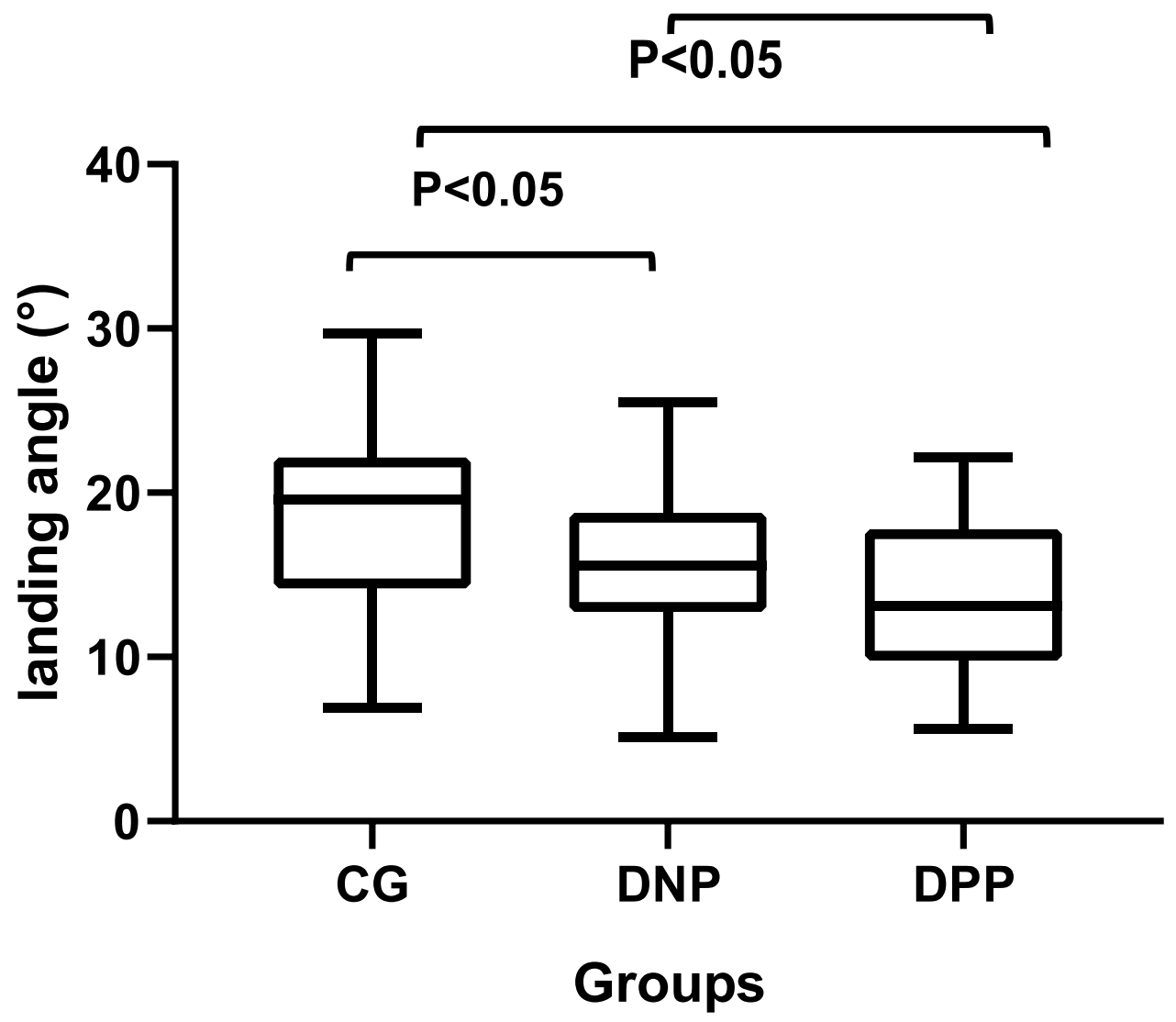

Figure 5 Mean of landing angle $\left({ }^{\circ}\right)$ in different groups.

Abbreviations: DPP, diabetic patients with peripheral neuropathy; DNP, diabetic patients without peripheral neuropathy; CG, control group.

and system (Gaitboter) have good repeatability and sensitivity. ${ }^{15}$ In addition, previous studies have confirmed that Gaitboter system is consistent, accurate and reliable compared with the three-dimensional gait analyzer. ${ }^{14}$ This study is the first to use this instrument in diabetic patients to analyze and study the gait of diabetic patients. The objective of this study is to explore the application value of this instrument in diabetes.

Before nerve damage, abnormal changes in electromyography (EMG) activity of lower limb muscles can be found in diabetic patients. The pathological changes of motor nerves are aggravated with neuropathy's progress, which is manifested by decreased muscle activity and delayed peak value. ${ }^{2}$ Muscle strength decline is a potential factor for falls, and walking speed can be used as an indicator of muscle strength decline. ${ }^{27}$ It has been reported that the walking speed of diabetic neuropathy patients decreased significantly. ${ }^{28}$ This paper showed that the walking speed with peripheral neuropathy is significantly less than those of the control group. Walking speed is a predictor of mortality. The improvement of walking speed can significantly reduce the mortality rate. ${ }^{29,30}$ Slow walking speed is a predictor of falls in the elderly. ${ }^{31}$ Some studies have confirmed that the risk of falling in the elderly with walking speed less than $0.70 \mathrm{~m} / \mathrm{s}$ is 1.5 times higher than that in the elderly with walking speed more than $0.70 \mathrm{~m} / \mathrm{s}$. Every $0.1 \mathrm{~m} / \mathrm{s}$ slowdown in walking speed increases the risk of falling by $7 \% .^{29}$ Other studies have found that the faster the walking speed, the higher will be the plantar pressure. ${ }^{32}$ The research on walking speed of diabetic patients is of great practical significance in preventing diabetic patients from falling and foot ulcer. Further studies are required to verify it.

This study also found that the foot progression angle, the stance phase and double-foot stance phase of diabetic patients tended to rise compared to the control group, but it did not show statistical significance. Diabetic patients 


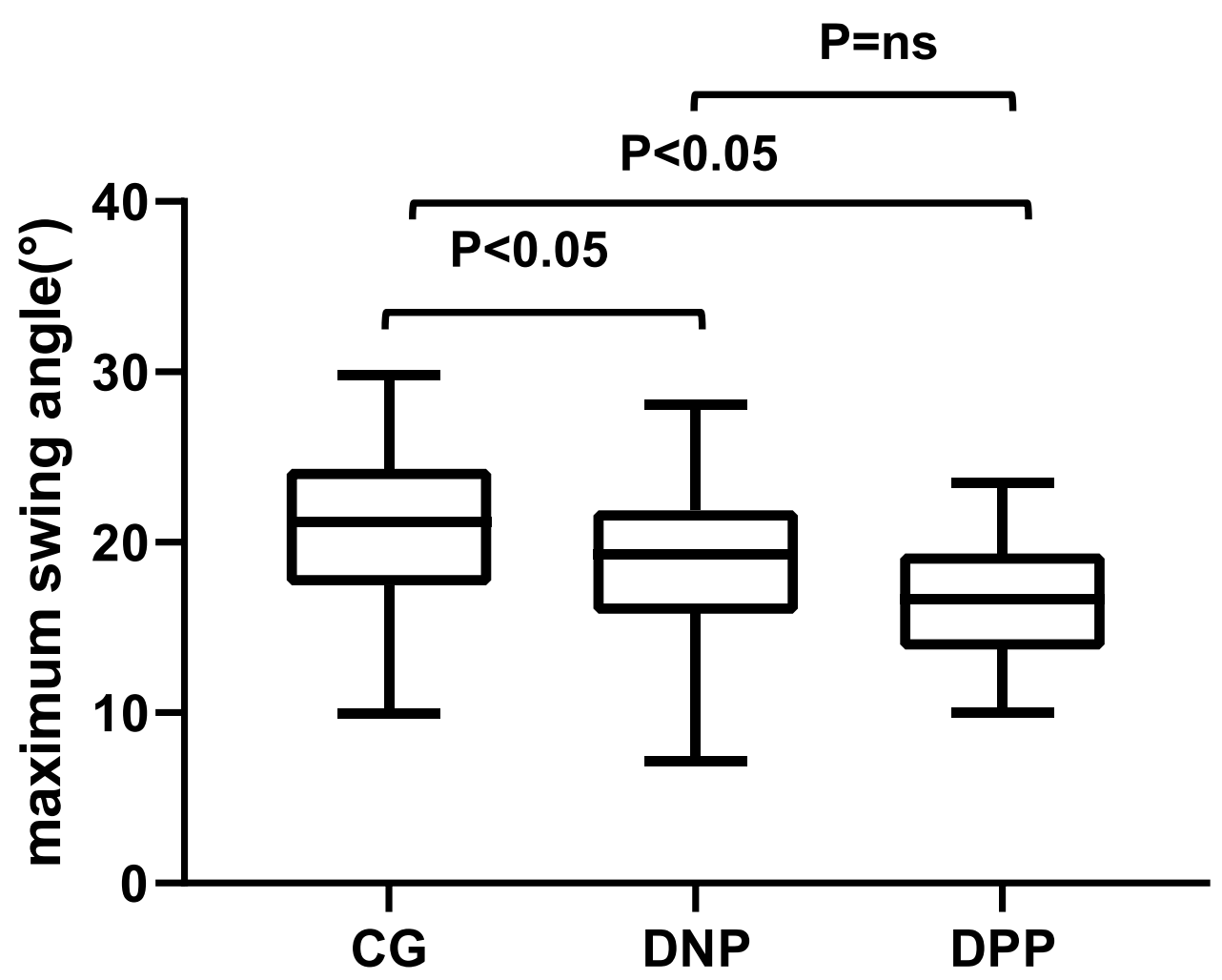

Figure 6 Mean of maximum swing angle $\left({ }^{\circ}\right)$ in different groups.

Abbreviations: DPP, diabetic patients with peripheral neuropathy; DNP, diabetic patients without peripheral neuropathy; CG, control group.

may correct their physical instability by prolonging stance phase and reducing the swing phase. Moreover, the longer the stance phase is, the longer will be plantar burden time, which may lead to an increase in the incidence of ulcers.

This study also found that the landing angle and the maximum swing angle of diabetic patients were lower than the control group, but there was no difference in the offground angle and the minimum swing angle. It reflects that the range of foot and ankle joint motion in diabetic patients decreases, and the movement is limited. Studies have shown that the lower extremity functional strength of diabetic patients is lower than that of healthy people of the same age group, regardless of the presence or absence of peripheral neuropathy. ${ }^{33}$ Sawacha et al did a study through three-dimensional gait analysis ${ }^{34}$ that the movement ability of trunk and lower limb joints of diabetic patients was lower than that of control group whether there was peripheral nerve damage or not, both static and dynamic, and the movement range of patients with peripheral neuropathy was reduced more significantly than the control group. The impaired proprioception of diabetic patients and the decreased strength of ankle dorsal flexor muscle and plantar flexor muscle lead to decreased balance ability in diabetic patients. ${ }^{35}$

In addition, it can be seen from the results that the landing angle and the maximum swing angle of diabetes patients without peripheral neuropathy tended to rise compared to diabetes patients with peripheral neuropathy. The walking speed of diabetes patients without peripheral neuropathy tended to rise compared to diabetes patients with peripheral neuropathy, and tended to decrease compared to the control group. These results did not show statistical significance. The reason is: considering that most of the subjects in the study were not-very-old adults with a notvery-long history of diabetes, so the patients with peripheral neuropathy had no serious nerve injury according to the previous studies. ${ }^{36,37}$ In further study, we will expand the sample size including more elder adults with a long history of diabetes and serious patient's condition of the subjects.

Our study had the limitation. The sample size of this article is not too large. Despite this limitation, we found that a wearable and portable gait analysis system (Gaitboter) can provide some quantifiable and easily 
obtained gait parameters for further scientific research in diabetes mellitus. We will expand the sample size in the future and conduct prospective research.

\section{Conclusions}

In conclusion, this study confirms that the wearable gait analysis system (Gaitboter) can identify abnormal gait in patients with type 2 diabetes, and provides a new device and method for diabetes-related gait research. Moreover, the system will help develop a huge gait database of type 2 diabetes mellitus. It has broad prospects for clinical application in the diabetes mellitus in the future.

\section{Acknowledgments}

The authors thank the role of all patients and support staff in performing the study.

\section{Funding}

This work is partly supported by Beijing Natural Science Foundation (4172058), 2025 Key Technology Innovation Program of Ningbo(2020Z014) and Henan Science and Technology Project (192102310218, 212102310557). The authors are grateful for these supports.

\section{Disclosure}

The authors declare that they have no conflicts of interest for this work.

\section{References}

1. Tesfaye S, Boulton AJ, Dyck PJ, et al; Toronto Diabetic Neuropathy Expert Group. Diabetic neuropathies: update on definitions, diagnostic criteria, estimation of severity, and treatments. Diabetes Care. 2010;33 (10):2285-2293. doi:10.2337/dc10-1303

2. Ricky W, Cristina DS, Andreja PP, et al. Effect of diabetic neuropathy severity classified by a fuzzy model in muscle dynamics during gait. J Neuroeng Rehabil. 2014;11:11. doi:10.1186/1743-0003-11-11

3. Sawacha Z, Guarneri G, Avogaro A, Cobelli C. A new classification of diabetic gait pattern based on cluster analysis of biomechanical data. $J$ Diabetes Sci Technol. 2010;4(5):1127-1138. doi:10.1177/ 193229681000400511

4. Skljarevski V, Malik RA. Clinical diagnosis of diabetic neuropathy. In: Veves A, Malik RA, editors. Diabetic Neuropathy: Clinical Management. 2. Totowa: Humana Press; 2007:275-292.

5. Boulton AJ. The diabetic foot: from art to science. The 18th Camillo Golgi lecture. Diabetologia. 2004;47(8):1343-1353. doi:10.1007/ s00125-004-1463-y

6. Petrofsky J, Lee S, Bweir S. Gait characteristics in people with type 2 diabetes mellitus. Eur J Appl Physiol. 2005;93(5-6):640-647. doi:10.1007/s00421-004-1246-7

7. Brach JS, Talkowski JB, Strotmeyer ES, Newman AB. Diabetes mellitus and gait dysfunction: possible explanatory factors. Phys Ther. 2008;88(11):1365-1374. doi:10.2522/ptj.20080016
8. Henderson AD, Wayne Johnson A, Ridge ST, et al. Diabetic gait is not just slow gait: gait compensations in diabetic neuropathy. J Diabetes Res. 2019;2019:9.

9. Carina P, Daniel P, Christopher N. Validity and repeatability of three in-shoe pressure measurement systems. Gait Posture. 2016;46:69-74. doi:10.1016/j.gaitpost.2016.01.026

10. Wang Z, Ji R. Estimate spatial-temporal parameters of human gait using inertial sensors. IEEE International Conference on Cyber Technology in Automation; 2015:1883-1888.

11. Lai DT, Begg R, Charry E, Palaniswami M, Hill K. Measuring toe clearance using a wireless inertial sensing device. In Proceedings of the 2008 IEEE International Conference on Intelligent Sensors, Sensor Networks and Information Processing (ISSNIP 2008); Sydney, NSW, Australia; December 15-18, 2008; 375-380.

12. Wang C, Wang XD, Long Z, Yuan J, Qian YL, Li JT. Estimation of temporal gait parameters using a wearable microphone-sensor-based system. Sensors. 2016;16(12):2167. doi:10.3390/s16122167

13. Wang C, Wang XD, Long Z, Yuan J, Qian YL, Li JT. Multimodal gait analysis based on wearable inertial and microphone sensors. 17 IEEE Ubiquitous Intelligence and Computing (UIC 2017).

14. Wang C, Wang XD, Long Z, et al. Estimation of spatial-temporal gait parameters based on the fusion of inertial and film-pressure signals. IEEE International Conference on Bioinformatics \& Biomedicine of 2018 (BIBM 2018).

15. Kong YQ, Liu MY, Wang C, Zhou LC, Wang XD. Sensibility and reliability of the gait analysis system based on the sound and motion sensors. Chin J Stroke. 2018;13(12):1262-1265.

16. Alam U, Riley DR, Jugdey RS, et al. Diabetic neuropathy and gait: a review. Diabetes Ther. 2017;8(6):1253-1264. doi:10.1007/s13300017-0295-y

17. Berti KG, Zimmet PZ. Definition diagnosis and classification of diabetes mellitus and its complications. Part 1: diagnosis and classification of diabetes mellitus provisional report of a WHO consultation. Diabet Med. 1998;15(7):539-553. doi:10.1002/(SICI) 1096-9136(199807)15:7<539::AID-DIA668>3.0.CO;2-S

18. Liu YX, Chen SZ, Zhou J, Guo RR, Tan HW, Yu YR. Early screening for diabetic peripheral neuropathy and its risk factors. Chin J Diabetes. 2016;24(6):536-539.

19. Cavanagh PR, Derr JA, Ulbrecht JS, Maser RE, Orchard TJ. Problems with gait and posture in neuropathic patients with insulin-dependent diabetes mellitus. Diabet Med. 1992;9 (5):469-474. doi:10.1111/j.1464-5491.1992.tb01819.x

20. Blake AJ, Morgan K, Bendall MJ, et al. Falls by elderly people at home: prevalence and associated factors. Age Ageing. 1988;17 (6):365-372. doi:10.1093/ageing/17.6.365

21. Bennetts CJ, Owings TM, Erdemir A, et al. Clustering and classification of regional peak plantar pressures of diabetic feet. $J$ Biomech. 2013;46(1):19-25. doi:10.1016/j.jbiomech.2012.09.007

22. Nagel A, Rosenbaum D. Vacuum cushioned removable cast walkers reduce foot loading in patients with diabetic mellitus. Gait Posture. 2009;30(1):11-15. doi:10.1016/j.gaitpost.2009.02.007

23. Allet L, Armand S, de Bie RA, et al. Clinical factors associated with gait alterations in diabetic patients. Diabet Med. 2009;26 (10):1003-1009. doi:10.1111/j.1464-5491.2009.02811.x

24. Esser P, Collett J, Maynard K, et al. Single sensor gait analysis to detect diabetic peripheral neuropathy: a proof of principle study. Diabetes Metab J. 2018;42(1):82-86. doi:10.4093/ dmj.2018.42.1.82

25. Rebula JR, Ojeda LV, Adamczyk PG, Kuo AD. Measurement of foot placement and its variability with inertial sensors. Gait Posture. 2013;38(4):974-980. doi:10.1016/j.gaitpost.2013.05.012

26. Li WX, Cao Y, Mc Z, et al. Analysis of foot biomechanics characteristic in 303 patients with type 2 diabetes mellitus. $J$ South Med Univ. 2016;36(10):1410-1416. 
27. Martinikorena I, Martínez-Ramírez A, Gómez M, et al. Gait variability related to muscle quality and muscle power output in frail nonagenarian older adults. J Am Med Dir Assoc. 2016;17 (2):162-167. doi:10.1016/j.jamda.2015.09.015

28. Allet L, Armand S, Golay A, et al. Gait characteristics of diabetic patients: a systematic review. Diabetes Metab Res Rev. 2008;24 (3):173-191. doi:10.1002/dmrr.809

29. Verghese J, Holtzer R, Lipton RB, et al. Quantitative gait markers and incident fall risk in older adults. J Gerontol a Biol Sci Med Sci. 2009;64A(8):896-901. doi:10.1093/gerona/glp033

30. Abellan van Kan G, Rolland Y, Houles M, et al. The assessment of frailty in older adults. Clin Geriatr Med. 2010;26(2):275-286. doi:10.1016/j.cger.2010.02.002

31. Pamoukdjian F, Paillaud E, Zelek L, et al. Measurement of gait speed in older adults to identify complications associated with frailty: a systematic review. J Geriatr Oncol. 2015;6(6):484-496. doi:10.1016/j.jgo.2015.08.006

32. Bumfield JM, Few CD, Mohamed OS, et al. The influence of walking speed and footwear on plantar pressures in older aduhs. Clin Biomech. 2004;19(1):78-84.
33. Vaz MM, Costa GC, Reis JG, et al. Postural control and functional strength in patients with type 2 diabetes mellitus with and without peripheral neuropathy. Arch Phys Med Rehabil. 2013;94 (12):2465-2470. doi:10.1016/j.apmr.2013.06.007

34. Sawacha Z, Gabriella G, Cristoferi G, et al. Diabetic gait and posture abnormalities: abiomechanical investigation through three dimensional gait analysis. Clin Biomech. 2009;24(9):722-728. doi:10.1016/j.clinbiomech.2009.07.007

35. Andreassen CS, Jakobsen J, Andersen H. Muscle weakness: a progressive late complication in diabetic distal symmetric polyneuropathy. Diabetes. 2006;55(3):3. doi:10.2337/diabetes.55.03.06.db05-1237

36. Ba Rbosa AP, Medina JL, Ramos EP, et al. Prevalence and risk factors of clinical diabetic polyneuropathy in a Portuguese primary health care population. Diabetes Metab. 2001;27(4 Pt 1):496-502.

37. Shaw JE, Hodge AM, Courten MD, et al. Diabetic neuropathy in Mauritius: prevalence and risk factors. Diabetes Res Clin Pract. 1998;42(2):131. doi:10.1016/S0168-8227(98)00100-4

Diabetes, Metabolic Syndrome and Obesity: Targets and Therapy

\section{Dovepress}

\section{Publish your work in this journal}

Diabetes, Metabolic Syndrome and Obesity: Targets and Therapy is an international, peer-reviewed open-access journal committed to the rapid publication of the latest laboratory and clinical findings in the fields of diabetes, metabolic syndrome and obesity research. Original research, review, case reports, hypothesis formation, expert opinion and commentaries are all considered for publication. The manuscript management system is completely online and includes a very quick and fair peer-review system, which is all easy to use. Visit http://www.dovepress.com/testimonials.php to read real quotes from published authors.

Submit your manuscript here: https://www.dovepress.com/diabetes-metabolic-syndrome-and-obesity-targets-and-therapy-journal 